\title{
Variation and asymmetry in host-symbiont dependence in a microbial symbiosis
}

\author{
Ewan J. A. Minter ${ }^{1}$, Chris D. Lowe², Megan E. S. Sørensen ${ }^{1}$, A. Jamie Wood ${ }^{3,4}$, Duncan D. Cameron \\ and Michael A. Brockhurst ${ }^{1 *}$ (D)
}

\begin{abstract}
Background: Symbiosis is a major source of evolutionary innovation and, by allowing species to exploit new ecological niches, underpins the functioning of ecosystems. The transition from free-living to obligate symbiosis requires the alignment of the partners' fitness interests and the evolution of mutual dependence. While symbiotic taxa are known to vary widely in the extent of host-symbiont dependence, rather less is known about variation within symbiotic associations.
\end{abstract}

Results: Using experiments with the microbial symbiosis between the protist Paramecium bursaria and the alga Chlorella, we show variation between pairings in host-symbiont dependence, encompassing facultative associations, mutual dependence and host dependence upon the symbiont. Facultative associations, that is where both the host and the symbiont were capable of free-living growth, displayed higher symbiotic growth rates and higher per host symbiont loads than those with greater degrees of dependence.

Conclusions: These data show that the Paramecium-Chlorella interaction exists at the boundary between facultative and obligate symbiosis, and further suggest that the host is more likely to evolve dependence than the algal symbiont.

\section{Background}

Symbiosis - the intimate living together of unlike organisms - is a major source of evolutionary innovation, providing interacting species with new functions and thus facilitating the evolution of complex life $[1,2]$. Symbioses are common in nature, and, by allowing species to exploit otherwise inaccessible ecological niches, underpin the diversity and functioning of natural ecosystems [3-5]. Yet understanding the origins and evolutionary stability of symbioses remains a major challenge for evolutionary biologists. The evolutionary transition from free-living to obligate symbiosis requires that the fitness interests of interacting species be aligned, and that the species evolve to become mutually dependent [6-10]. However, while famous examples of obligate symbiotic partnerships exist, many symbioses are facultative wherein species retain the ability to survive in the free-living state $[11,12]$. Comparative evolutionary analysis suggests that

\footnotetext{
*Correspondence: m.brockhurst@sheffield.ac.uk

${ }^{1}$ Department of Animal and Plant Sciences, University of Sheffield, Alfred

Denny Building, Western Bank, Sheffield S10 2TN, UK

Full list of author information is available at the end of the article
}

this variation among lineages in their degree of host-symbiont dependence is at least partially explained by the types of benefits exchanged between symbiotic partners and the mode of symbiont inheritance, with mutual dependence being more common in vertically-inherited, nutritional symbioses [13]. These macroevolutionary patterns cannot reveal, however, the extent of variation in host-symbiont dependence available to natural selection within symbioses, nor the potential for asymmetries in dependence among partners in a symbiosis.

Photosynthetic endosymbioses (photosymbioses), typically between eukaryotic algae and animal or protist hosts, are a classic example of widespread and ecologically important symbiosis $[5,12,14]$ and therefore represent a useful model system for understanding evolutionary transitions in symbiosis. Photosymbioses are typically based upon the reciprocal exchange of nutrients in the form of fixed carbon from symbiont to host, and nitrogen compounds from host to symbiont [15]. Photosymbioses vary widely in their degree of host-symbiont dependence, from ancient and obligate organelles (e.g. primary, secondary, and tertiary plastids in eukaryotic algae, see Keeling [16]), 
to facultative symbioses where symbiotic partners are also able to survive in the free-living state (e.g. Symbiodinium and anthozoan corals [17, 18]). Across the extant eukaryotic tree of life, transitions from facultative to obligate photosymbiosis have occurred independently a number of times $[16,19,20]$, yet facultative photosymbioses are arguably both more common and more diverse $[12,21]$. Little is known, however, about variation in host-symbiont dependence within facultative photosymbioses and the ecological drivers selecting for maintenance of the facultative habit.

The microbial photosymbiosis between the host Paramecium bursaria - a heterotrophic ciliate- and the symbiont Chlorella sp. - a green alga- is a tractable model system [22-27] where the fitness effects of symbiosis relative to free-living can be directly quantified [28]. The $P$. bursaria-Chlorella $(\mathrm{Pb}-\mathrm{C})$ symbiosis is widespread in shallow freshwater habitats, and is primarily based upon provision of nitrogen compounds from host heterotrophy to the symbiont, and of maltose and oxygen derived from symbiont photosynthesis to the host [29-32]. The $\mathrm{Pb}-\mathrm{C}$ symbiosis has evolved multiple times, such that, whilst each $\mathrm{Pb}-\mathrm{C}$ strain contains a clonal population of Chlorella, multiple origins of symbiotic lineages occur across the Chlorella clade [33, 34]. Within the Chlorella clade, C. vulgaris are found in both the free-living and symbiotic states, whereas $C$. variabilis is more typically associated with symbiosis [35]. We have previously shown for a single $\mathrm{Pb}-\mathrm{C}$ pairing that the fitness effects of symbiosis are environmentally context dependent and highly asymmetric: For hosts, symbiosis is costly in the dark but becomes increasingly beneficial with increasing irradiance, whereas, for symbionts, symbiosis is not beneficial and becomes increasingly costly with increasing irradiance [28]. Hosts exert tight control over symbiont load (i.e., the number of symbionts per host cell), regulating symbiont number in relation to light to maximise the benefit-to-cost ratio of symbiosis $[28,36]$. Accordingly, symbiont load peaks at low light levels but is reduced both in the dark, where symbionts are not beneficial, and at high light levels, where per symbiont benefits are highest [28]. Given the inherent conflict between these symbiotic partners, and the strong environmental context dependence of the fitness effects of symbiosis, we hypothesise that selection to retain free-living growth should be stronger for Chlorella than $P$. bursaria due to the asymmetries in the fitness benefits of symbiosis.

Here we experimentally investigate natural variation in host-symbiont dependence by comparing free-living versus symbiotic growth among five $\mathrm{Pb}-\mathrm{C}$ pairings. We report variation in both the fitness effects of symbiosis and host-symbiont dependence between $\mathrm{Pb}-\mathrm{C}$ pairings. Among the five $\mathrm{Pb}-\mathrm{C}$ pairings, we observed fully facultative associations, an association displaying mutual dependence, and associations in which hosts alone displayed dependence. Notably, symbiotic growth rates were higher in $\mathrm{Pb}-\mathrm{C}$ pairings that retained the fully facultative lifestyle and maintained higher symbiont loads. Our data therefore show that $\mathrm{Pb}-\mathrm{C}$ pairings vary in the degree of host-symbiont dependence, and suggest that Paramecium is more likely to evolve dependence than Chlorella.

\section{Materials \& methods}

\section{Paramecium strains and culturing conditions}

Experiments were performed using five Paramecium bursaria strains along with their naturally occurring Chlorella symbionts. These $\mathrm{Pb}-\mathrm{C}$ pairings are designated 186b, HA1, HK1, CT39, and Dd1. 186b (CCAP 1660/18) was obtained from the Culture Collection for Algae and Protozoa (Oban, Scotland) and isolated in the UK, whilst the remaining four pairings were all obtained from the Paramecium National Bio-Resource Project (Yamaguchi, Japan) and were all isolated in Japan. Further details of the $\mathrm{Pb}-\mathrm{C}$ pairings used are provided in Additional file 1: Table S1. All experiments were performed by culturing in bacterized Protozoan Pellet Media (PPM, Carolina Biological Supply, NC, USA) which was made to a concentration of $0.66 \mathrm{~g} \mathrm{~L}^{-1}$ with Volvic natural mineral water, and inoculated approximately $20 \mathrm{~h}$ prior to use with Serratia marcescens from frozen glycerol stocks. All stock cultures were maintained at $25{ }^{\circ} \mathrm{C}$ with $50 \mu \mathrm{E} \mathrm{m}{ }^{-}$ ${ }^{2} \mathrm{~s}^{-1}$ of light and a 14:10 L:D cycle. Stock cultures were maintained by batch culture, where cultures were diluted by half every $2-3$ weeks with fresh bacterized PPM. Unless otherwise stated, experiments were performed under the same culture conditions.

\section{Symbiotic and apo-symbiotic host growth rates in response to light}

Growth rates of hosts were compared across a light gradient and in the presence (symbiotic) or absence (apo-symbiotic) of Chlorella symbionts. Apo-symbiotic cell cultures were established by treating symbiotic cells with a combination of paraquat $\left(10 \mu \mathrm{g} \mathrm{mL}^{-1}\right)$ and cyclohexamide $\left(10 \mu \mathrm{g} \mathrm{mL}^{-1}\right)$ and exposing the cells to high light intensities $\left(>50 \mu \mathrm{E} \mathrm{m}^{-2} \mathrm{~s}^{-1}\right)$ for a period of between four and seven days, until host cells were visibly symbiont free. Apo-symbiotic cell cultures were verified by monitoring the colour of host cells on the microscope, and observing that re-greening by Chlorella did not occur over three weeks.

Both symbiotic and apo-symbiotic $P$. bursaria cells were washed and concentrated using sterile Volvic and re-suspended in bacterized PPM. Cells were acclimated to $50 \mu \mathrm{E} \mathrm{m}^{-2} \mathrm{~s}^{-1}$ light for two days before being washed once again, re-suspended in fresh bacterized PPM. Cells were then acclimated to their treatment light condition 
$\left(0,1.5,3,6,12,25, \& 50 \mu \mathrm{E} \mathrm{m}^{-2} \mathrm{~s}^{-1}\right)$ for five days before being washed and re-suspended in bacterized PPM at a target cell density of approximately 350 cells $\mathrm{mL}^{-1}$. To estimate growth rates, cell densities were determined at 0 , 24 , and $48 \mathrm{~h}$ by fixing $350 \mu \mathrm{L}$ of each cell culture, in triplicate, in $1 \% v / \mathrm{v}$ glutaraldehyde in 96 -well flat bottomed micro-well plates. Images of each well after settling were recorded using a Nikon D600 camera mounted to an inverted microscope through a $4 \times$ objective lens. Cell counts for each well were recorded using an automated image analysis macro in Image J v1.50i [37].

\section{Free living Chlorella growth}

Free-living algal cultures were established in triplicate by washing $10 \mathrm{~mL}$ of stock culture in approximately $200 \mathrm{~mL}$ of sterile Volvic on an $11 \mu \mathrm{m}$ nylon mesh. Host cells retained by the mesh were re-suspended in $1.5 \mathrm{~mL}$ Volvic and ultra-sonicated using a Fisherbrand Q500 Sonicator (Fisher Scientific, NH, USA), at a power setting of $20 \%$ for $10 \mathrm{~s}$. Ultra-sonication resulted in lysis of host cells (confirmed by visual inspection) and release of symbionts into the surrounding media. Algal symbionts were separated from host cell lysate by centrifugation, re-suspended in $5 \mathrm{~mL}$ Bold's Basal Medium (BBM) [38], and cultured in $30 \mathrm{~mL}$ glass tubes under the same conditions as for host stock cultures but with the addition of shaking at $130 \mathrm{rpm}$. The dynamics of these populations were tracked for five days. Cell densities were estimated each day using a CytoFLEX $S$ flow cytometer (Beckman Coulter Inc., CA, USA), and manually gating Chlorella events for each individual sample using CytExpert2.0 (Beckman Coulter Inc., CA, USA). Specifically, Chlorella cells were distinguished from other particles on the basis of their fluorescence and size characteristics, which were initially determined by visual inspection of a subset of the flow cytometry data.

\section{Host symbiont load in response to light}

$P$. bursaria cells with symbionts were washed and concentrated using sterile Volvic and re-suspended in bacterized PPM. Cells were evenly split into 28 microcosms each containing $5 \mathrm{~mL}$ of bacterized PPM and microcosms were randomly assigned to one of seven light treatment groups $(n=4)$. Microcosms were acclimated to their light treatment $\left(0,1.5,3,6,12,25, \& 50 \mu \mathrm{E} \mathrm{m}^{-2} \mathrm{~s}^{-1}\right)$ for approximately 6 days prior to flow cytometry analysis.

Host symbiont loads were estimated using a CytoFLEX $S$ flow cytometer (Beckman Coulter Inc., CA, USA) by measuring the intensity of chlorophyll fluorescence for individual $P$. bursaria cells (excitation $488 \mathrm{~nm}$, emission $690 / 50 \mathrm{~nm}$ ). Data are presented as relative fluorescence, and are calibrated against 8-peak beads, to reduce variation between samples run in separate sessions.

\section{Data analysis}

All statistical analyses were performed in $\mathrm{R}$ v.2.3.4 ( $\mathrm{R}$ Core Development Team, 2016). The raw data analysed here is provided in Additional file 2, and further details of the fitted statistical models are provided in Additional file 3 . Host growth rates were analysed treating light as either a continuous variable or a factor (the results of both analyses were qualitatively similar). In the first analysis, strain, symbiont presence/absence, and light were treated as factors. In the second analysis, since the relationship between growth and light differed markedly for symbiotic and apo-symbiotic hosts, we analysed these responses separately to detect strain-specific differences in growth using linear and non-linear regression for apo-symbiotic and symbiotic responses, respectively. Symbiotic host growth responses were modelled as:

$$
r=\frac{r_{\max }\left(L-p^{\prime}\right)}{k+\left(L-p^{\prime}\right)}
$$

where $r$ is growth rate at a given light intensity $(L), r_{\max }$ is the light dependent maximum growth rate, $k$ is the half saturation constant and $p$ is the threshold light concentration (i.e. light concentration when growth is zero). Free living symbiont growth rates were analysed by One Way ANOVA.

Host symbiont loads were analysed by non-linear regression and non-linear mixed effects models (NLME) with the function

$$
\phi=\frac{a\left(L-l^{\prime}\right)}{b+(L-l \prime)^{c}}
$$

where $\phi$ equals the mean host symbiont load (relative units of chlorophyll host ${ }^{-1}$ ) at a given light intensity $(L)$, $a, b, c$, and $l$ are parameters.

\section{Results}

To examine variation in the effect of symbionts on host growth, we grew multiple independent strains of $P$. bursaria across a light gradient, both with and without symbionts. Growth rates for hosts with symbionts increased with light, whereas growth rates for hosts without symbionts were unaffected by light levels (light by symbiosis interaction, $F_{1,213}=69.3, P<0.001$ ), and the effect of symbionts on host growth varied between host strains (strain by symbiosis interaction, $\mathrm{F}_{3,213}=3.5, P=0.009$ ). To further understand these patterns, growth responses of hosts with and without symbionts were analysed separately (Additional file 3). For all host strains, symbiotic host growth rates were either zero or negative in the dark and increased as a function of light, in most host strains this response was asymptotic reaching a maximum growth rate at high light levels (Fig. 1). Host strains HA1 and 186b with symbionts had the highest 


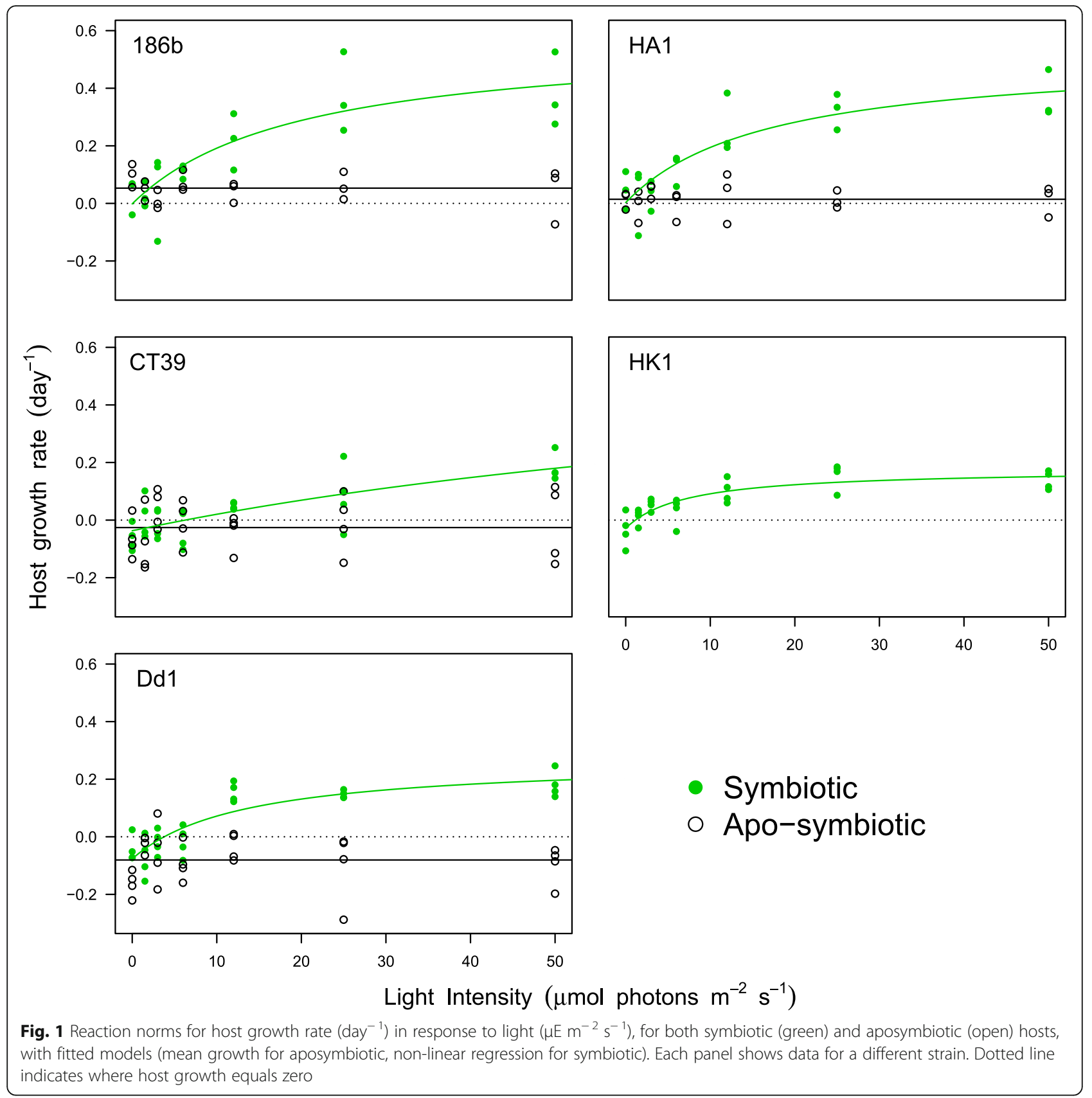

maximum growth rates $\left(r_{\max }\right)$ and were significantly higher than in host strain HK1 with symbionts (twosample t-tests: HK1 vs HA1, $t=3.104, P=0.039$; HK1 vs 186b, $t=3.097, P=0.036$ ). Host growth rates without symbionts varied between symbiont-free host strains (ANOVA, $\mathrm{F}_{3,94}=15.0, P<0.001$ ), from low (HA1 \& 186b) to negative growth rates (CT39 \& Dd1), and did not respond to light (ANOVA, $\mathrm{F}_{6,88}=0.57, P=0.757$ ). For one host strain, HK1, hosts without symbionts did not survive. These data suggest that host strains varied both in the benefit derived from symbiosis and in their dependence upon symbionts for growth and survival.
To estimate survival of algal symbionts in the free-living state, Chlorella were isolated from their host and grown for one week in $50 \mu \mathrm{E} \mathrm{m} \mathrm{m}^{-2} \mathrm{~s}^{-1}$ light and population densities measured daily. Algal strains varied in their free-living growth rate (Fig. 2, ANOVA, $\mathrm{F}_{5,12}=$ $767, \mathrm{P}<0.001$ ). Four algal strains displayed positive growth rates, whereas algae isolated from Dd1 were unable to grow in the free-living state (Fig. 2). Taken together with the data for free-living host growth rates, these data suggest that whereas some $\mathrm{Pb}-\mathrm{C}$ pairings were facultative, wherein both the host and symbiont were capable of free-living (186b and HA1), other $\mathrm{Pb}-\mathrm{C}$ 


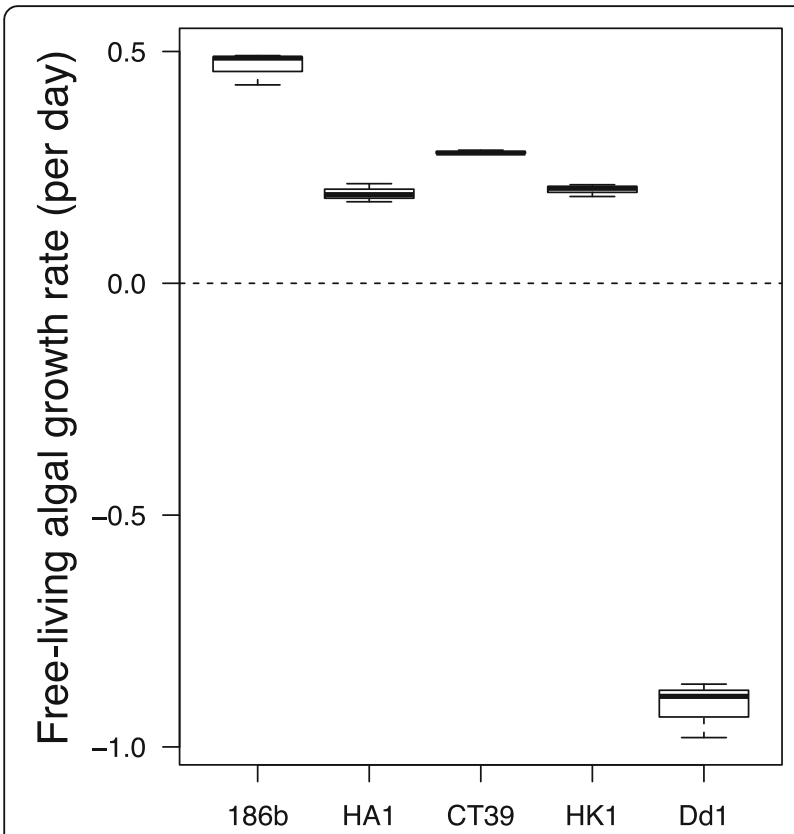

Fig. 2 Growth rate of extracted Chlorella symbionts in 7-day cultures grown in Bold's Basal Medium immediately following mechanical liberation from Paramecium bursaria hosts. Boxes show median and ranges for three independent culture replicates, dotted line indicates zero growth

pairings displayed some degree of dependence. For example, both the host and symbiont from the strain Dd1 were mutually dependent (i.e., unable to sustain free-living growth), while in CT39 and HK1 the symbionts were capable of free-living but the hosts were not, suggesting host dependency upon symbionts.

We previously showed that hosts tightly regulate symbiont load in relation light level to maximise the benefit-to-cost ratio of symbiosis [28]. To test whether host control varied among $\mathrm{Pb}-\mathrm{C}$ pairings and was related to the degree of host-symbiont dependence we measured the per host symbiont load of each $\mathrm{Pb}-\mathrm{C}$ pairing across a light gradient. Consistent with our previous finding, across all hosts, symbiont loads were lowest in the dark, peaked at low light intensities $\left(2-8 \mu \mathrm{E} \mathrm{m}^{-2} \mathrm{~s}^{-1}\right)$, and declined to intermediate levels at high light intensities (Fig. 3). While this pattern of symbiont load was broadly consistent among hosts, we did observe minor variations in the estimated parameters of the fitted curves (NLME, $X_{6}^{2}=118, P<0.001$; see Additional file 3$)$. Specifically, host strain $186 \mathrm{~b}$ had a higher symbiont load than HK1 independent of light level (i.e. parameter $a$ ). Peak symbiont load occurred at lower light intensities in host strains 186b and Dd1 than HK1 and CT39 (i.e. parameter $l$ ), potentially suggesting differences in the light environment to which the $\mathrm{Pb}-\mathrm{C}$ pairings were adapted in nature. Host strains Dd1 and HA1 reduced symbiont load at high light intensities to a greater extent than host

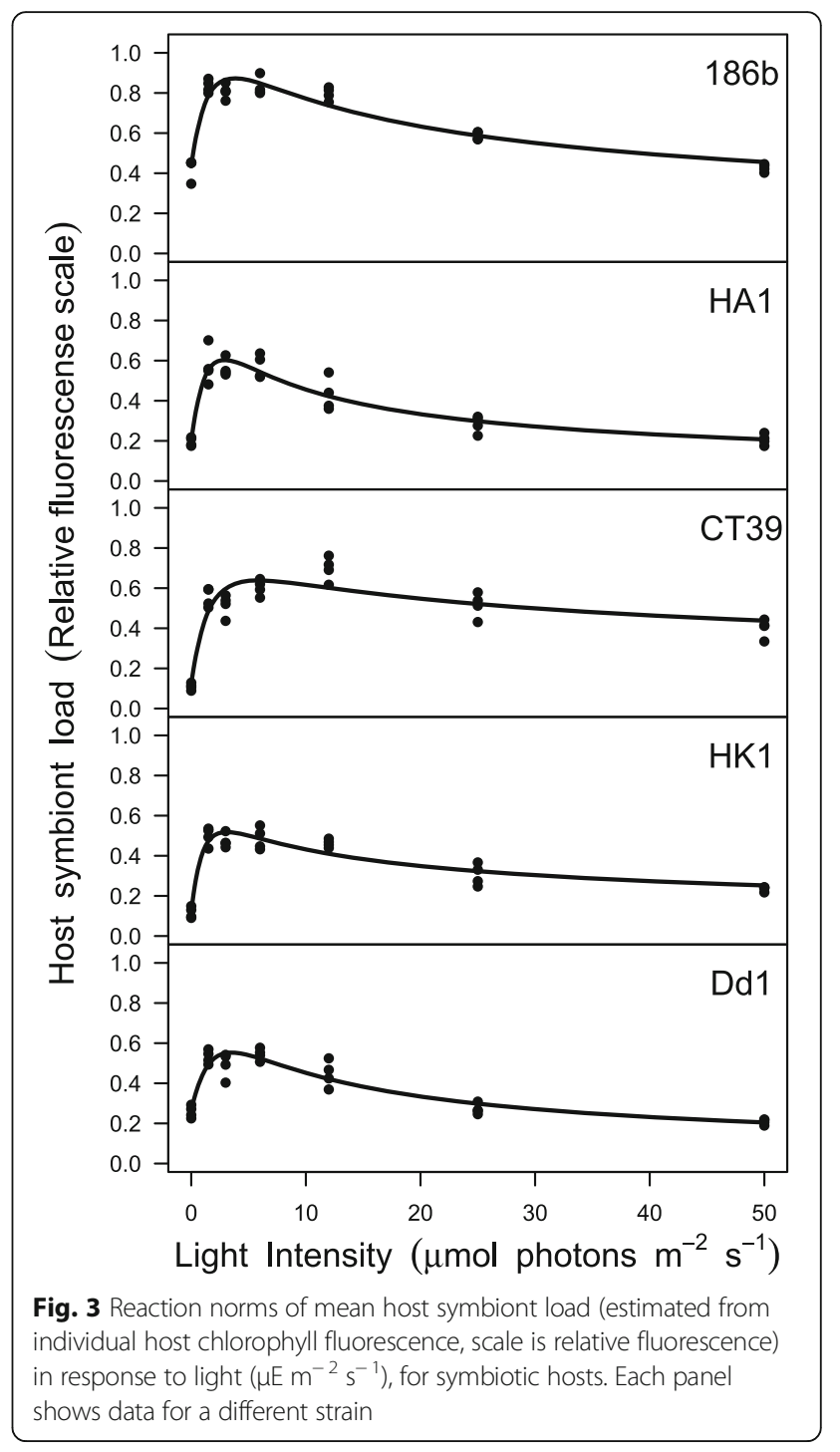

strain CT39 (i.e. parameter $c$ ), suggesting variation in the intensity of host regulation of symbiont load. These data suggest that host control is a broadly conserved trait across $P$. bursaria, but show no clear association between host control parameters and host-symbiont dependence, except that symbiont load was highest in the most facultative host (186b) and lowest in the host least able to survive without its symbionts (HK1).

\section{Discussion}

The transition from facultative to obligate symbiosis, and thus the evolution of mutual dependence constitutes a major evolutionary transition in individuality [8,9], and underpins the evolution of cellular organelles such as chloroplasts [1]. The evolutionary transition to mutual dependence requires there to be variation in host-symbiont dependence available for natural selection to act upon, and for mutual dependence to be associated with higher 
symbiotic fitness [6-10]. Using experiments with the microbial photosymbiosis between the ciliate host, $P$. bursaria, and the green alga, Chlorella sp., we demonstrate variation in host-symbiont dependence ranging from $\mathrm{Pb}-\mathrm{C}$ pairings that are fully facultative to those that display either mutual dependence or dependence of hosts upon symbionts. Thus the P. bursaria-Chlorella interaction appears to exist on the boundary between facultative and obligate symbiosis. Moreover, since symbiotic growth rates of facultative $\mathrm{Pb}-\mathrm{C}$ pairings were higher than those showing greater degrees of dependence-indeed the host HK1 which was unable to survive without symbionts showed the lowest symbiotic growth rate-it seems likely that facultative symbiosis may be favoured by selection. Interestingly, this is consistent with the distribution of symbiotic strains across the predominantly free-living Chlorella clade [34], which suggests repeated transitions from free-living to symbiosis and a long evolutionary history of its association with $P$. bursaria being facultative. Furthermore, $\mathrm{Pb}-\mathrm{C}$ pairings that were more recently isolated from natural populations (186b and HA1 were isolated in 2006 and 2010, respectively; Additional file 1: Table S1), were more facultative than those with longer histories of laboratory culture (HK1 and Dd1 were isolated in 1990 and 1995, respectively; Additional file 1: Table S1). This could suggest that host-symbiont dependence is a derived trait among lab-adapted $\mathrm{Pb}-\mathrm{C}$ pairings, whereas in natural populations the facultative state is more common, however more extensive studies of natural populations will be required to test this hypothesis.

Dependence was more commonly observed in P. bursaria than in Chlorella, possibly suggesting an asymmetry in selection for dependence between the host and the symbiont in this system. This would be consistent with our previous work, which showed that this is an exploitative symbiotic interaction, wherein hosts benefit more than symbionts from engaging in symbiosis [28]. This underlying conflict between host and symbiont would be expected to select for the retention of free-living ability, particularly in the symbiont. The fitness benefit of symbiosis to hosts increases with increasing light intensity and with decreasing availability of heterotrophic food [28], suggesting that selection for dependence in hosts is likely to be environmentally context dependent. We would predict therefore that $P$. bursaria should be more likely to evolve dependence on their symbionts in high light, low food habitats, but less likely in low light, high food habitats, or in environments that are highly variable in terms of light intensity and/or food availability. Indeed, in variable environments the facultative nature of the photosymbiosis may allow for partner-switching whereby hosts could acquire locally-adapted symbionts to promote their invasion of new habitats. Experimental tests of the effects of partner-switching on host-symbiont phenotype in this system are required to disentangle the contributions of host genotype, symbiont genotype and their interaction to host-symbiont fitness and local adaptation.

We observed similar responses among hosts in their regulation of symbiont load across light gradients. Consistent with our previous data [28] and a mathematical model of this system [36], we observed that symbiont load per host peaked at low light levels. This occurs because hosts adjust symbiont number to maximise the benefit-to-cost ratio of symbiosis [36]. In the dark, hosts reduce their symbiont load as their maintenance is costly and they provide no benefit to host growth through photosynthesis. At very low light intensities, hosts need many symbionts in order to gain a growth benefit, which albeit costly in terms of demand for nitrogen leads to a peak in symbiont load. As light increases, the per symbiont benefit to hosts increases and so hosts need fewer symbionts to provide the same photosynthetic output, allowing hosts to reduce their $\mathrm{N}$ costs. Above a given light level, the per-symbiont benefit saturates leading to an asymptotic relationship between symbiont load and light. The response of symbiont load to light was broadly conserved among our host strains, and our empirical estimates of this trait closely matched the theoretical predictions in Dean et al. [36]. Minor variations in the parameters of the fitted curves were observed but were not associated with variation in dependency, with the exception that symbiont load was highest in the fastest growing and most facultative host (186b) and lowest in the host that was slowest growing and least able to survive without its symbionts (HK1). This suggests that while all host strains have the ability to control symbiont load, an overall higher symbiont load favoured faster symbiotic growth, whereas lower symbiont loads may have evolved in more highly dependent associations, presumably to minimise the costs of symbiosis.

\section{Conclusions}

Comparative evolutionary analysis suggests that hostsymbiont dependence varies widely between symbiotic lineages across the tree of life [13]. Data from the study presented here show that the degree of host-symbiont dependence also varies within symbiotic partnerships, and asymmetrically for hosts and symbionts. Where symbiosis is based upon exploitation, as here, our data suggest that the evolution of dependence is less likely in the exploited symbiotic partner, in this case, Chlorella.

\section{Additional files}

Additional file 1: Table S1. Details of the Paramecium-Chlorella strains used in this study. (DOCX $12 \mathrm{~kb}$ )

Additional file 2: The raw data that was presented and analysed in the manuscript. (XLSX $42 \mathrm{~kb}$ ) 
Additional file 3: Model outputs for the statistical analyses presented in the manuscript. (DOCX $46 \mathrm{~kb}$ )

\section{Abbreviations}

BBM: Bold's Basal Medium; L:D: Light:Dark; PPM: Protozoan Pellet Media

\section{Acknowledgements}

We are grateful to Richard Law and Andrew Dean for discussions. We are grateful to Karen Hogg from the Technology Facility in the Department of Biology at the University of York for assistance with the flow cytometry.

\section{Funding}

This work was funded by grant NE/K011774/2 from the Natural Environment Research Council, UK to MAB, CDL, DDC, and AJW, a studentship from the Biotechnology and Biological Sciences Research Council, UK to MESS (BB/ M011151/1), a Philip Leverhulme Prize (PLP-2014-242) from the Leverhulme Trust to MAB, and start-up funding from the University of Sheffield. DDC is supported by a Royal Society University Research Fellowship (UF090328). The funders had no role in the design of the study, the collection, analysis and interpretation of data, or the writing of the manuscript.

\section{Availability of data and materials}

All data generated or analysed during this study are included in this published article and its additional files.

\section{Authors' contributions}

EJAM, CDL, AJW, DDC and MAB designed the study; EJAM and MESS performed the experiments; EJAM analysed the data; EJAM, DDC and MAB drafted the manuscript; all authors contributed to the writing the final draft; all authors have read and approved the manuscript.

\section{Ethics approval and consent to participate}

Not applicable.

\section{Consent for publication}

Not applicable.

\section{Competing interests}

The authors declare that they have no competing interests

\section{Publisher's Note}

Springer Nature remains neutral with regard to jurisdictional claims in published maps and institutional affiliations.

\section{Author details}

'Department of Animal and Plant Sciences, University of Sheffield, Alfred Denny Building, Western Bank, Sheffield S10 2TN, UK. ${ }^{2}$ Centre for Ecology and Conservation, University of Exeter, Penryn TR10 9FE, UK. ${ }^{3}$ Department of Biology, University of York, York YO10 5DD, UK. ${ }^{4}$ Department of Mathematics, University of York, York YO10 5DD, UK.

\section{Received: 23 February 2018 Accepted: 29 June 2018}

\section{Published online: 09 July 2018}

\section{References}

1. Smith JM, Szathmary E. The major transitions in evolution. OUP Oxford. 1997:361.

2. Oliver KM, Degnan PH, Burke GR, Moran NA. Facultative symbionts in aphids and the horizontal transfer of ecologically important traits. Annu Rev Entomol. 2010;55(1):247-66.

3. Muscatine L, Porter JW. Reef corals: mutualistic symbioses adapted to nutrient-poor environments. Bioscience. 1977 Jul 1:27(7):454-60.

4. Smith SE, Read DJ. Mycorrhizal Symbiosis. Academic Press. 2010:815

5. Mitra A, Flynn KJ, Burkholder JM, Berge T, Calbet A, Raven JA, et al. The role of mixotrophic protists in the biological carbon pump. Biogeosciences. 2014 Feb 20;11(4):995-1005.

6. Bennett GM, Moran NA. Heritable symbiosis: the advantages and perils of an evolutionary rabbit hole. Proc Natl Acad Sci. 2015 Aug 18;112(33):10169-76.

7. Cavalier-Smith T. Symbiogenesis: mechanisms, evolutionary consequences, and systematic implications. Annu Rev Ecol Evol Syst. 2013;44(1):145-72.
8. Estrela S, Kerr B, Morris JJ. Transitions in individuality through symbiosis. Curr Opin Microbiol. 2016 Jun;31:191-8.

9. West SA, Fisher RM, Gardner A, Kiers ET. Major evolutionary transitions in individuality. Proc Natl Acad Sci. 2015 Aug 18;112(33):10112-9.

10. Kiers ET, West SA. Evolving new organisms via symbiosis. Science. 2015 Apr 24;348(6233):392-4

11. Simon J-C, Carré S, Boutin M. Prunier-Leterme N, Sabater-Muñoz B, Latorre a, et al. host-based divergence in populations of the pea aphid: insights from nuclear markers and the prevalence of facultative symbionts. Proc $R$ Soc Lond B Biol Sci. 2003 Aug 22;270(1525):1703-12.

12. Stoecker DK, Johnson MD, de VC, Not F. Acquired phototrophy in aquatic protists. Aquat Microb Ecol. 2009 Nov 24;57(3):279-310.

13. Fisher RM, Henry LM, Cornwallis CK, Kiers ET, West SA. The evolution of host-symbiont dependence. Nat Commun 2017 Jul 4;8:ncomms15973.

14. Pfeffer PE, Douds DD, Bécard G, Shachar-Hill Y. Carbon uptake and the metabolism and transport of lipids in an arbuscular mycorrhiza. Plant Physiol. 1999 Jun 1;120(2):587-98.

15. Yellowlees D, Rees TAV, Leggat W. Metabolic interactions between algal symbionts and invertebrate hosts. Plant Cell Environ. 2008 May 1;31(5):679-94.

16. Keeling PJ. The number, speed, and impact of plastid endosymbioses in eukaryotic evolution. Annu Rev Plant Biol. 2013;64:583-607.

17. Baker AC. Flexibility and specificity in coral-algal Symbiosis: diversity, ecology, and biogeography of Symbiodinium. Annu Rev Ecol Evol Syst. 2003;34(1):661-89.

18. Banaszak AT, Iglestas-Prieto R, Trench RK. Scrippsiella Velellae Sp. Nov. (peridiniales) and Gloeokinium Viscum Sp. Nov. (phytodiniales), dinoflagellate symbionts of two hydrozoans (cnidiaria)1,2. J Phycol. 1993 Aug 1:29(4):517-28.

19. Baurain D, Brinkmann H, Petersen J, Rodríguez-Ezpeleta N, Stechmann A, Demoulin V, et al. Phylogenomic evidence for separate Acquisition of Plastids in Cryptophytes, haptophytes, and Stramenopiles. Mol Biol Evol. $2010 \mathrm{Jul}$ 1;27(7):1698-709.

20. Yokobori S, Kurabayashi A, Neilan BA, Maruyama T, Hirose E. Multiple origins of the ascidian-Prochloron symbiosis: molecular phylogeny of photosymbiotic and non-symbiotic colonial ascidians inferred from $18 \mathrm{~S}$ rDNA sequences. Mol Phylogenet Evol 2006 Jul 1;40(1):8-19.

21. Decelle J, Colin S, Foster RA. Photosymbiosis in marine planktonic Protists. In: Marine Protists. Springer, Tokyo; 2015. p. 465-500.

22. Fujishima M. Endosymbionts in Paramecium. Springer Science \& Business Media. 2009:260

23. Kodama Y, Fujishima M. Four important cytological events needed to establish endosymbiosis of symbiotic Chlorella sp. to the alga-free Paramecium bursaria. Jpn. J Protozool. 2011;44(1):1.

24. Kodama Y, Fujishima M. Cell division and density of symbiotic Chlorella variabilis of the ciliate Paramecium bursaria is controlled by the host's nutritional conditions during early infection process. Environ Microbiol. 2012 Oct 1;14(10):2800-11.

25. Tanaka M, Murata-Hori M, Kadono T, Kawano T, Yamada T, Kosaka T, et al. Complete elimination of endosymbiotic algae from Paramecium bursaria and its confirmation by diagnostic PCR. Acta Protozool. 2002;41(3):255-62

26. Tanaka M, Miwa I. Significance of photosynthetic products of symbiotic Chlorella to establish the endosymbiosis and to express the mating reactivity rhythm in Paramecium bursaria. Zool Sci. 1996 Oct 1;13(5):685-92.

27. Kodama Y, Fujishima M. Symbiotic Chlorella sp. of the ciliate Paramecium bursaria do not prevent acidification and lysosomal fusion of host digestive vacuoles during infection. Protoplasma. 2005 Jul 8; 225(3-4):191-203.

28. Lowe CD, Minter EJ, Cameron DD, Brockhurst MA. Shining a light on exploitative host control in a photosynthetic endosymbiosis. Curr Biol. 2016 Jan 4:26(2):207-11.

29. Ziesenisz E, Reisser W, Wiessner W. Evidence of de novo synthesis of maltose excreted by the endosymbiotic Chlorella from Paramecium bursaria. Planta. 1981 Dec 1;153(5):481-5.

30. Reisser W. The metabolic interactions between Paramecium bursaria Ehrbg. And Chlorella spec. In the Paramecium bursaria-symbiosis. II. Symbiosis-specific properties of the physiology and the cytology of the symbiotic unit and their regulation (author's transl). Arch Microbiol. 1976 Dec;111(1-2):161-70.

31. Brown JA, Nielsen PJ. Transfer of photosynthetically produced carbohydrate from endosymbiotic Chlorellae to Paramecium bursaria*. J Protozool. 1974 Oct 1;21(4):569-70 
32. Kato $Y$, Ueno S, Imamura N. Studies on the nitrogen utilization of endosymbiotic algae isolated from Japanese Paramecium bursaria. Plant Sci. 2006 Mar; 170(3):481-6.

33. Zagata P, Greczek-Stachura M, Tarcz S, Rautian M. The evolutionary relationships between endosymbiotic green algae of Paramecium bursaria Syngens originating from different geographical locations. Folia Biol (Praha). $2016 \operatorname{Jan} 29 ; 64(1): 47-54$.

34. Hoshina R, Imamura N. Multiple origins of the symbioses in Paramecium bursaria. Protist. 2008 Jan 7;159(1):53-63.

35. Hoshina R, Iwataki M, Imamura N. Chlorella variabilis and Micractinium reisseri sp. nov. (Chlorellaceae, Trebouxiophyceae): Redescription of the endosymbiotic green algae of Paramecium bursaria (Peniculia, Oligohymenophorea) in the 120th year. Phycol Res. 2010 Jul 1:58(3):188-201.

36. Dean AD, Minter EJA, Sørensen MES, Lowe CD, Cameron DD, Brockhurst MA, Wood AJ. Host control and nutrient trading in a photosynthetic symbiosis. J Theor Biol. 2016 Apr 6;405(1):82-93.

37. Schneider CA, Rasband WS, Eliceiri KW. NIH image to ImageJ: 25 years of image analysis. Nat Methods. 2012;9:671-5.

38. Stein JR. Handbook of phycological methods: physiological and biochemical methods, vol. 2: Cambridge University Press; 1973.

Ready to submit your research? Choose BMC and benefit from:

- fast, convenient online submission

- thorough peer review by experienced researchers in your field

- rapid publication on acceptance

- support for research data, including large and complex data types

- gold Open Access which fosters wider collaboration and increased citations

- maximum visibility for your research: over $100 \mathrm{M}$ website views per year

At BMC, research is always in progress.

Learn more biomedcentral.com/submissions 